\title{
IN SITU AUV SURVEY ADAPTATION USING THROUGH-THE-SENSOR SONAR DATA
}

\author{
David P. Williams, Arjan Vermeij, Francesco Baralli, Johannes Groen, Warren L.J. Fox \\ NATO Undersea Research Centre, Viale San Bartolomeo 400, 19126 La Spezia (SP), Italy
}

\begin{abstract}
An algorithm for the in situ adaptation of the survey route of an autonomous underwater vehicle (AUV) equipped with side-looking sonars is proposed. The algorithm immediately exploits the through-the-sensor data that is collected during the mission in order to ensure that quality data is collected everywhere in the area of interest. By introducing flexibility into the survey of the AUV, various limitations of pre-planned surveys are overcome. Experimental results demonstrate the benefit of the proposed approach in terms of higher area coverage in shorter mission times. The signal processing required by the algorithm is fast and computationally efficient such that real-time implementation is feasible. As proof, the proposed adaptive survey approach was implemented on an AUV and executed during a recent live scientific experiment at sea using real, in situ measured data. Results from this experiment are also shown.
\end{abstract}

Index Terms - Data collection, in situ adaptation, sonar, image quality, AUV

\section{INTRODUCTION}

The high-resolution imaging of underwater environments afforded by synthetic aperture sonar (SAS) [1] has proven useful in a wide range of applications. Thanks to breakthroughs in marine robot technology, the sonar data is now often collected by an autonomous underwater vehicle (AUV) equipped with two side-looking sonars, one on the port side and one on the starboard side.

These sensors image in directions orthogonal to the direction in which the AUV travels. Because of the geometry of the problem, a "dead zone" - from directly below the AUV out to a certain range on either side - between the two sonar swaths will lack sonar coverage.

The standard AUV survey plan used in practice [2] consists of a series of equidistant parallel tracks - often referred to as a "lawnmower pattern" - such that the sonar swaths of consecutive tracks interleave, resulting in sonar coverage for the entire area of interest.

The implicit assumption made in this purely pre-planned approach is that quality sonar coverage will be achieved consistently to a certain range on either side of the AUV's trackcenterline. However, if this coverage is underestimated (so the area of seabed interrogated on consecutive tracks overlaps), the coverage rate decreases, causing more time and resources to be expended to survey the mission area. If this coverage is overestimated, no usable sonar data will be collected for certain portions of the mission area, resulting in gaps in coverage.

Designing a survey based on assumed coverage is a real problem because data collected at sea with an AUV is rarely flawless [3]. For example, sonar image quality at long range is often poor because of signal attenuation or excessive undesired vehicle motion (caused by currents) that can result in defocused imaging.

A rigid pre-planned survey route is suboptimal because it has no flexibility to adapt to the environmental variability encountered during the data collection process at sea if the $a$ priori coverage assumptions that determine the set of tracks are not satisfied.

Therefore, in this work, we develop an algorithm to intelligently collect side-looking sonar data by adapting, in situ, the AUV survey route based on the characteristics observed in the through-the-sensor data being collected to ensure that the data quality is sufficiently high.

By immediately adapting the survey route in situ, the need to re-deploy the AUV (to fill in gaps in the data coverage) is obviated. In turn, the time and costs of the data-collection mission are significantly reduced. The generality of the algorithm makes it applicable to a diverse set of researchers who rely on sonar data.

Previous work related to adaptive underwater path planning has not focused on ensuring the quality of the data being collected, as we do here. Instead, the main body of work has been interested in surveying only a subset of an area after an event of interest (such as reefs [4]) has been detected, rather than performing an exhaustive search of an area. Other work has adopted adaptive strategies to minimize errors due to temporal evolution of the ocean during the survey [5], or to reach a mission-specified destination while minimizing energy costs [6]. The work involving dense, exhaustive surveys of an area with an AUV has tended to focus on navigation correction and localization [7].

The remainder of this paper is organized as follows. The proposed strategy for performing adaptive data collection based on the quality of the through-the-sensor sonar data is outlined in Sec. 2. Experimental results of the algorithm, 
including its execution at sea on a real AUV are shown in Sec. 3. Concluding remarks and directions for future work are provided in Sec. 4.

\section{ADAPTIVE SURVEY ALGORITHM}

\subsection{Overview}

This work presents an algorithm to adaptively determine, in situ, the optimal placement of AUV survey tracks that will ensure quality sonar data is collected over the entire area of interest. The peak correlation of consecutive ping returns, as a function of range, is used to quantify the sonar image quality. To predict the area that would be covered with quality data by a hypothetical survey track, a full Bayesian model of this correlation quantity is continuously updated during the mission based on the ongoing performance of the sonars. The objective function that then drives the track selection is defined in terms of the expected gain in coverage and the transit cost of each potential track.

\subsection{Image Quality}

A sonar image with good image quality is one in which objects and seabed features appear more or less as they would when observed under the ideal operating conditions of the sonar system.

Synthetic aperture sonar (SAS), which provides an orderof-magnitude improvement in resolution over simple (real aperture) side-scan sonar data, works by coherently summing received sonar signals, or ping-returns, of overlapping elements in the array. The peak correlation between successive pings at a given range (i.e., distance from the sonar) provides a measure of the success of the SAS processing — and in turn, the image quality - as this quantity is directly proportional to the signal-to-noise ratio (SNR) [8].

Although full SAS processing is a computationally intensive procedure, the ping-to-ping correlation can be easily calculated onboard an AUV in real-time — with minimal computational power - as data is collected.

Analysis of a detection algorithm on a large data set of sonar images containing 480 man-made targets [9] indicated that the performance was noticeably affected by the image quality when the peak correlation was less than $\tau_{\rho}=2 / 3$. Therefore, in this work, we deem data to be of insufficient quality below this threshold.

\subsection{Quality Coverage}

For a given track, let $\rho(r, u)$ be the peak correlation value between consecutive pings at range $r$ on sonar side $u \in$ \{port, starboard\}, averaged over all pings associated with the track.

Let $\nu(r, u)$ be the historical success rate of collecting quality sonar data during the mission. This success rate will essentially be a running tabulation of the proportion of tracks at each range that resulted in quality data (i.e., $\rho(r, u) \geq \tau_{\rho}$ ) during the mission, and will be updated via a full Bayesian model after each track is executed.

The likelihood of coverage success (a binary variable) achieved during a track for a particular $(r, u)$-pair can be considered to follow a Bernoulli distribution with probability $\nu$ of success. The prior expressing our a priori beliefs about the sonar's performance is assumed to follow a Beta distribution, $\mathcal{B}(\alpha, \beta), p(\nu)=\nu^{\alpha-1}(1-\nu)^{\beta-1} C(\alpha, \beta)$, where $\alpha>0$ and $\beta>0$ are shape parameters and $C$ is a normalization constant to ensure the total probability integrates to unity. Specifically, the prior we employ is $\nu(u, r) \sim \mathcal{B}(\alpha=1, \beta=1)$ for $r \in\left[k_{\min }, k_{\max }\right]$ (and otherwise, outside these valid ranges, 0 ), where $k_{\min }$ and $k_{\max }$ are the nominal operation limits of the sonar; this Beta prior is simply a uniform distribution over $(0,1)$.

Because the Beta prior is a conjugate prior for a Bernoulli likelihood, the posterior distribution over $\nu$ is another Beta distribution, with parameters $\alpha=Q+1$ and $\beta=T-Q+1$, where $Q$ is the number of tracks that successfully achieved quality coverage at the given $(r, u)$, out of $T$ total tracks (i.e., attempts).

That is, $Q(r, u)=\sum_{i=1}^{T} Q_{i}(r, u)$, where $Q_{i}(r, u)$ is a binary variable indicating whether the $i$-th of $T$ tracks in the present mission resulted in quality coverage at range $r$ from sonar side $u$ (i.e., whether $\rho(r, u) \geq \tau_{\rho}$ during the $i$-th track).

The expected value of a random variable $\nu$ following a Beta distribution with parameters $\alpha$ and $\beta$ is $\mathbb{E}[\nu]=\frac{\alpha}{\alpha+\beta}$. Therefore, the expected value of the posterior for successful quality coverage is

$$
\mathbb{E}[\nu(r, u)]=\frac{Q(r, u)+1}{T+2} .
$$

This posterior expectation is used in the objective function calculation for determining the next track to execute.

By tying the expected future performance in the remainder of the mission to the historical in situ coverage performance of the sonars, the anticipated coverage by a hypothetical survey track can be more accurately predicted.

\subsection{New Track Selection}

Given an area of seabed to be surveyed, a series of parallel tracks will be executed over that area. The proposed algorithm adaptively determines, in situ, the specific locations of those tracks based on the through-the-sensor data collected.

The mission area is discretized into a set of non-overlapping swaths. Associated with each swath, $s_{i}$, is a binary coverage variable, $\gamma\left(s_{i}\right)$. (For a given track, there exists a unique mapping, based on simple geometry, from each $(r, u)$-pair to some particular $s_{i}$.) If $\rho(r, u) \geq \tau_{\rho}$ after executing a track, the corresponding swath coverage variable, $\gamma\left(s_{i}\right)$, is set to unity. Then the utility of executing each potential new track is calculated. 
The utility of running a new track, $t_{i}$, having just executed track $t_{j}$, is simply the difference between the expected benefit and the cost, $U\left(t_{i} \mid t_{j}\right)=\left[B\left(t_{i}\right)-C\left(t_{i} \mid t_{j}\right)\right] \mathbf{1}_{B\left(t_{i}\right)>0}$, where the indicator function $\mathbf{1}_{B\left(t_{i}\right)>0}$ is included to ensure that only tracks with positive benefits are considered. The track $t_{i}$ (that improves the coverage) for which the utility is maximized is then selected as the next track to be executed. This process is repeated until $\gamma\left(s_{i}\right)=1 \forall s_{i}$ (i.e., all coverage variables are unity), indicating that quality sonar data has been collected for the entire mission area.

The benefit of running a particular track, $t_{i}$, as the next track is defined to be the weighted sum of the expected coverage improvement, $B\left(t_{i}\right)=\sum_{r} \sum_{u} \mathbb{E}[\nu(r, u)](1-\gamma(r, u))$, where $\gamma(r, u)$ is the binary coverage quantity for the swath of seabed at range $r$ on sonar side $u$, computed with respect to the centerline of track $t_{i} ; \mathbb{E}[\nu(r, u)]$ is obtained from (1).

The cost of running a particular track, $t_{i}$, is a function of the transit distance required to reach the new track from the vehicle's present location. Let $d\left(t_{j}, t_{i}\right)$ be the distance between the most-recently executed track, $t_{j}$, and a potential track $t_{i}$. Let $\omega$ denote twice the turning radius of the vehicle. The cost of executing track $t_{i}$, having just executed track $t_{j}$, is defined to be $C\left(t_{i} \mid t_{j}\right)=\left(d\left(t_{i}, t_{j}\right) / \omega\right)^{2}-\left(2 d\left(t_{i}, t_{j}\right) / \omega\right)+1$, which is the equation of a parabola with a vertex at $(d, C)=$ $(\omega, 0)$, indicating that the transit cost is a minimum (namely, zero) when two tracks are separated by twice the turning radius of the vehicle.

\section{EXPERIMENTAL RESULTS}

In May 2011, the NATO Undersea Research Centre (NURC) conducted the ARISE sea trial in the Mediterranean Sea off the coast of Italy. High-resolution sonar data was collected during this trial with the MUSCLE AUV, which is equipped with a $300 \mathrm{kHz}$ sonar with a $60 \mathrm{kHz}$ bandwidth that can achieve image resolution of approximately $3 \mathrm{~cm}$.

The proposed adaptive track-spacing algorithm was implemented on the MUSCLE AUV and executed at sea during this trial. To demonstrate the algorithm, we will show an example simulation result, followed by the result of the algorithm in action at sea.

\subsection{Simulation Experiments}

First, experiments were conducted for a simulated, rectangular mission area that was $3.82 \mathrm{~km} \times 1.71 \mathrm{~km}$. The maximum range at which quality sonar data was achieved for each track was determined using real measured sonar data collected at sea (in a different area).

The tracks of the pre-planned survey approach are designed optimally in the sense that the fewest number of tracks must be executed if sonar swath coverage is always achieved from $k_{\min }=40 \mathrm{~m}$ to $k_{\max }=150 \mathrm{~m}$ away from the AUV in both the port and starboard directions; in this case, 10 tracks

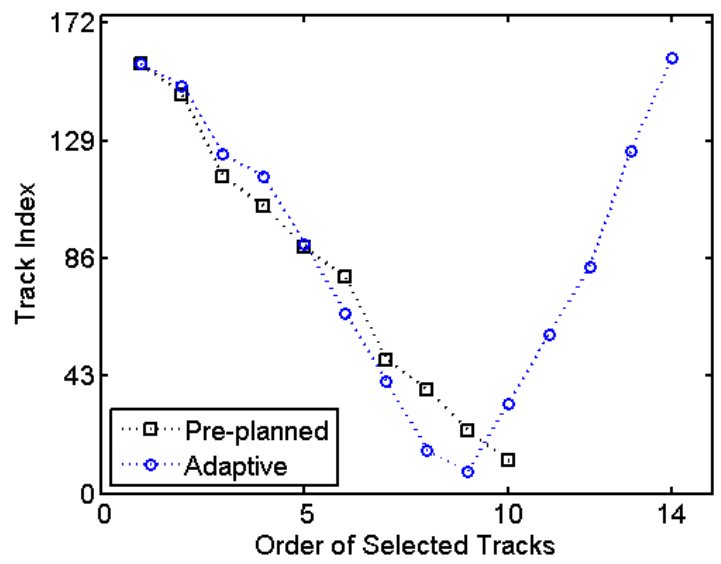

Fig. 1. Locations of the tracks selected by each algorithm.

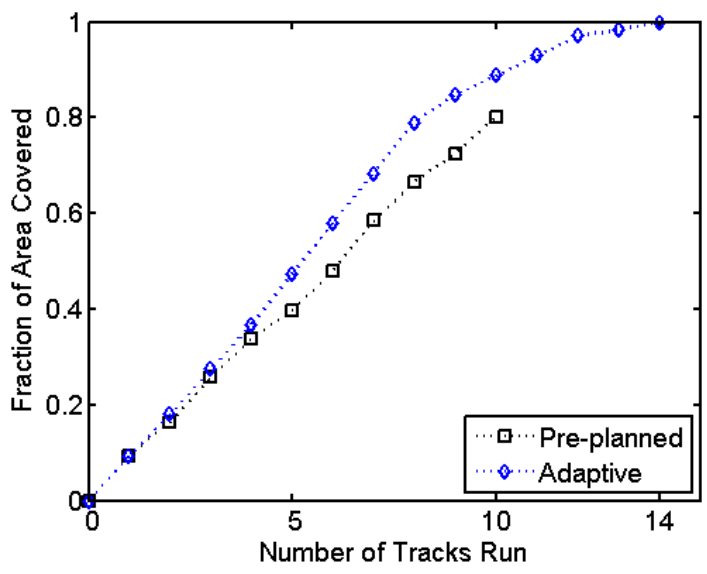

Fig. 2. Progression of the fraction of the mission area for which quality data was collected by each algorithm.

would be required. The adaptive approach selects its tracks based on the algorithm in Sec. 2. The locations of the tracks selected by each method is shown in Fig. 1.

The progression of the fraction of the mission area for which quality data was collected, as a function of the number of tracks executed by each method, is shown in Fig. 2. Because of poor quality data at long range, the pre-planned survey achieved quality coverage over only $80.12 \%$ of the mission area. In contrast, the proposed approach adapted its survey based on the data collected in situ to ensure quality coverage was obtained over $100 \%$ of the mission area. It can be observed in Fig. 1 how the adaptive method selected extra tracks ( $c f$. tracks 10-14) to cover areas for which the image quality had been poor on the first pass through the mission area, thereby effectively filling in the gaps in coverage. 


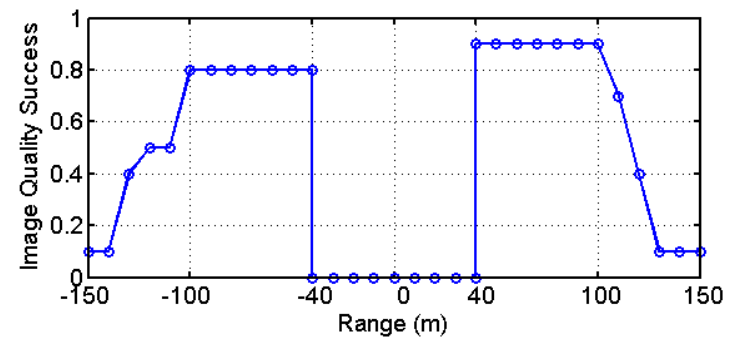

Fig. 3. The historical image quality success for the port and starboard sonars at the end of the adaptive mission at sea (after 8 tracks).

\subsection{At-Sea Experiments}

A rectangular area of interest, of size $500 \mathrm{~m} \times 1300 \mathrm{~m}$, near Tellaro, Italy was the focus of both pre-planned and adaptive surveys during the ARISE sea trial. The survey tracks were oriented parallel to the shorter pair of sides of the area.

The pre-planned survey consisted of 13 tracks such that the coverage of consecutive tracks heavily overlapped with each other (a common strategy employed so that two views of each seabed location are obtained). An adaptive survey based on the algorithm presented in Sec. 2 was also executed over the same mission area. Eight tracks, adaptively determined at sea based on the through-the-sensor data being collected onboard the AUV during the mission, were executed (in complete autonomy) before the mission was manually aborted for safety reasons.

During this mission, the sonar data quality at long range was often poor beyond approximately $100 \mathrm{~m}$. To demonstrate this quantitatively, the success rate from (1), for good quality data being collected as a function of range, at the end of the adaptive mission is shown in Fig. 3.

The coverage of the mission area, for each of the two approaches, is shown as a function of survey time in Fig. 4. The adaptive strategy achieved higher coverage more quickly than the purely pre-planned approach. If the adaptive survey was not manually aborted prematurely, complete coverage should have been achieved.

\section{CONCLUSION}

A new algorithm permitting adaptive AUV surveys at sea based on through-the-sensor data collected in situ was presented. The flexible, adaptive approach ensures that good quality data is collected over the entire area of interest without requiring post-mission analysis and AUV re-deployment. Results from a preliminary demonstration of the algorithm operating in real time at sea were also presented. More extensive experiments at sea will be conducted in an upcoming sea trial.

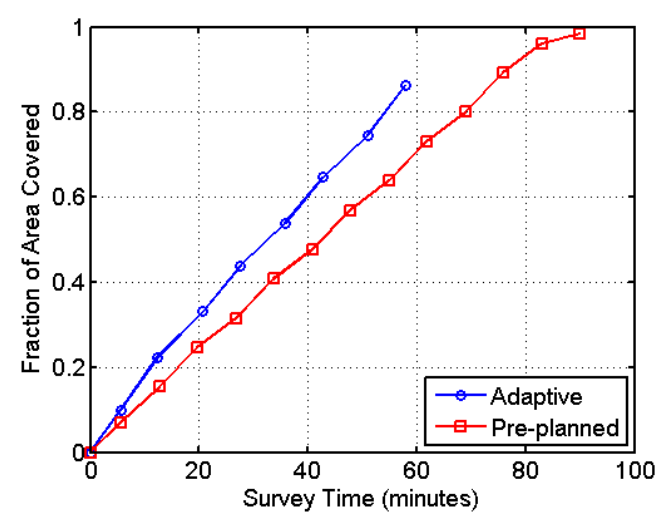

Fig. 4. Progression of the fraction of the mission area for which quality data was collected by each algorithm as a function of survey time. The end of each track is indicated by a marker on the plot.

\section{REFERENCES}

[1] M. Hayes and P. Gough, "Broad-band synthetic aperture sonar," IEEE J. Oceanic Eng., vol. 17, no. 1, pp. 80-94, 1992.

[2] C. Warren, "A technique for autonomous underwater vehicle route planning," IEEE J. Oceanic Eng., vol. 15, pp. 199-204, 1990.

[3] R. Hansen, H. Callow, T. Sæbø, and S. Synnes, "Challenges in seafloor imaging and mapping with synthetic aperture sonar," in Proc. Euro. Conf. SAR, 2010.

[4] A. Bender, S. Williams, O. Pizarro, and M. Jakuba, "Adaptive exploration of benthic habitat using Gaussian processes," in Proc. IEEE OCEANS, 2010.

[5] J. Bellingham and J. Willcox, "Optimizing AUV oceanographic surveys," in Proc. IEEE Symp. AUVs Tech., 1996, pp. 391-398.

[6] A. Alvarez, A. Caiti, and R. Onken, "Evolutionary path planning for autonomous underwater vehicles in a variable ocean," IEEE J. Oceanic Eng., vol. 29, no. 2, pp. 418-429, 2004.

[7] I. Ruiz, S. Raucourt, Y. Petillot, and D. Lane, "Concurrent mapping and localization using sidescan sonar," IEEE J. Oceanic Eng., vol. 29, no. 2, pp. 442-456, 2004.

[8] S. Synnes, R. Hansen, and T. Sæbø, "Assessment of shallow water performance using interferometric sonar coherence," in Proc. UAM, 2009.

[9] J. Groen, E. Coiras, and D. Williams, "Detection rate statistics in synthetic aperture sonar images," in Proc. UAM, 2009, pp. 367-374. 